\title{
Pediatric Neurosurgical Fellowships
}

As our young speciality develops and strives for excellence we are concerned about our beginnings but must not loose sight of our future. If excellence in the care of children with diseases of the nervous system is our primary goal, the preparation of the pediatric neu-rosurgeons should be our first concern.

Most of us practicing pediatric surgical subspeciali-ties are the product of a system designed, appropriately, primarily for the care of adults. This process begins in medical school where embryology has been reduced to a brief overview and little is taught about the physiology of the child. It continues through our internship which usually includes little or no pediatrics. This is further compounded by the fact that $30 \%$ of training programs do not meet the meager recommendations of the Board of Neurosurgery. Many of the other programs do not supply training at a level that most pediatric neurosur-geons would feel is adequate to ensure routine neurosurgical care of the child.

In the design of a fellowship, exposure to the other pediatric subspecialities is essential. These include pediatric neurology, neuroradiology and pathology. Interaction with pediatric surgery, pediatric urology, orthopedics and plastic surgery must be more than by casual consultation. 
An area of particular concern is the training of academic pediatric neurosurgeons. We must foster, both at the clinical and basic science level, the study of the diseases that threaten children. A fellowship must, at the least, allow the opportunity for research and optimally require the development of research skills.

Beyond our obligation to contribute to the body of knowledge in our speciality, we owe this preparation to those we train. Many of the fellows will ultimately head pediatric neurosurgical sections. The lack of an understanding of the scientific method, how to develop a laboratory, or how to obtain funding places a section head at a significant disadvantage.

To accomplish competence in all of these areas in 1 year is unlikely. It may be that many of these skills should be acquired in the postfellowship practice of pediatric neurosurgery. Although not a popular view, I feel 1 year is not adequate preparation and would favor a more extended period which includes a sequence of rotations to other pediatric subspecialities as well as evidence of academic performance.

Our aim should not be the minimum of training needed or the number we can produce but rather what kind of pediatric neurosurgeon would best advance our infant speciality toward excellence.

David G. McLone, MD, PhD 